\title{
Hypsipyla grandella em Mogno (Swietenia macrophylla): Situação Atual e Perspectivas
}

\author{
Alexandre Mehl Lunz ${ }^{(1)}$; Marcílio José Thomazini(2); Maria Carolina Blassioli Moraes( ${ }^{(3)}$; Edinelson José Maciel Neves(2); \\ Telma Fatima Coelho Batista(4); Juliana Degenhardt(2); Lindáurea Alves de Sousa ${ }^{(1)}$; Orlando Shigueo Ohashi( ${ }^{(4)}$
}

\begin{abstract}
(1)Embrapa Amazônia Oriental, Travessa Dr. Enéas Pinheiro, s/nº, Marco, CEP 66085-100, Belém-PA. E-mails: amehl@cpatu.embrapa.br, lindas@amazon.com; (2)Embrapa Florestas, Estrada da Ribeira, Km 111, C.P. 319, CEP 83411-000, Colombo-PR. E-mails: marcilio@cnpf.embrapa.br, eneves@cnpf.embrapa.br, juliana@cnpf.embrapa.br; ${ }^{(3)}$ Embrapa Recursos Genéticos e Biotecnologia, Parque Estação Biológica-PqEB,Av. W5 Norte (final), C.P. 02372, CEP 70770-917, Brasília-DF. E-mail: mcbmorae@cenargen.embrapa.br; ${ }^{(4)}$ Instituto de Ciências Agrárias, Universidade Federal Rural da Amazônia (ICA/UFRA), Av. Tancredo Neves, 2501, Montese, CEP 66077-530, Belém-PA. E-mails: telma.batista@ufra.edu.br, shigueo@amazon.com
\end{abstract}

\begin{abstract}
Resumo - A madeira de mogno, Swietenia macrophylla King, é valorizada no mercado internacional por sua beleza e durabilidade para fins nobres, como mobiliários e decoração. Quaisquer tentativas de plantios comerciais dessa espécie de modo a reduzir a pressão exercida pela sua exploração seletiva invariavelmente fracassam devido à incidência da broca do ponteiro do mogno, Hypsipyla grandella (Zeller). Embora alguns trabalhos tenham obtido relativo sucesso em apresentar técnicas de controle sob aspectos pontuais, ainda não foi estabelecida uma estratégia de manejo integrado que seja prática, econômica e eficiente para $\mathrm{H}$. grandella no Brasil. Este trabalho tem como objetivo rever e atualizar informações sobre o inseto e seu hospedeiro, além de indicar as áreas de pesquisa mais promissoras para o seu controle em plantios de S. macrophylla no País. As estratégias de controle de $H$. grandella mais recomendadas são o uso da resistência da planta, manejo silvicultural, uso de semioquímicos e controle biológico, nas quais há necessidade de incentivos a pesquisas de longo prazo. Ensaios de resistência natural de procedências de $S$. macrophylla devem ser incentivados, devido às extensas áreas com reservas naturais no Brasil.
\end{abstract}

Termos para indexação: Broca do ponteiro do mogno, broca das Meliaceae, Cedrela odorata, manejo integrado de pragas.

\section{Hypsipyla grandella in Mahogany (Swietenia macrophylla): Current Situation and Perspectives}

\begin{abstract}
The mahogany wood, Swietenia macrophylla King, is valued in the international trade market for its beauty and durability for noble uses like furniture and decoration. Any attempt of commercial plantation of this specie to diminish the pressure made by its selective exploration fail due to the incidence of the mahogany shoot borer, Hypsipyla grandella (Zeller). Although some works have had relative success in presenting control techniques over the punctual aspects, a strategy of economic, an economic strategy for efficient and integrated management practice was not established yet for $H$. grandella in Brazil. This study objective is to update data about the insect and its host based in the most recent studies made in Brazil, besides to indicate the most prominent research areas for its control in S. macrophylla plantations in the Country. The most recommended control's strategies for $H$. grandella are the host plant resistance use, silvicultural management, semiochemicals and biological control, in which exist the need of income to the long term researches. Natural resistance tests of $S$. macrophylla procedences must be encouraged due to Brazil's wide natural resource areas.
\end{abstract}

Index terms: Mahogany shoot borer, Meliaceae's borer, Cedrela odorata, integrated pest management.

\section{Introdução}

O plantio de árvores da família Meliaceae com fins comerciais nos trópicos é inviável devido à ação das larvas do gênero oligófago pantropical Hypsipyla Zeller (Lepidoptera: Pyralidae) (GRIJPMA, 1976; MAHROOF et al., 2002), que contempla várias espécies.
A intensidade do ataque varia conforme a região e a essência utilizada. São reconhecidas duas espécies principais: $H$. grandella (Zeller), na região tropical da América Latina e sul do Estado da Flórida, e H. robusta (Moore), no sul e sudeste da Ásia, Austrália e no oeste e leste da África (NAIR, 2007). 
No Brasil, a ocorrência de $H$. grandella em árvores de mogno (Swietenia macrophylla King) torna impraticável a sua implantação em cultivos maciços comerciais. $\mathrm{O}$ principal dano do inseto consiste na destruição do broto terminal em mudas e árvores novas devido à entrada $\mathrm{e}$ escavação de galerias pelas larvas (GRIJPMA, 1976). Os sintomas do ataque são representados pela exsudação de goma e serragem, provenientes da atividade larval, pela presença de folhas secas em meio à folhagem verde, e pela emissão de novos ponteiros a cada ataque consecutivo, que também são atacados (SILVA, 1985; GRIFFITHS, 2000; OHASHI et al., 2002). As galerias descendentes promovem a seca e, eventualmente, a queda da região apical. O crescimento e a formação de um tronco retilíneo, que é o grande objetivo comercial da cultura, são seriamente prejudicados (GRIJPMA, 1976; OHASHI et al., 2000) com perda em altura de $35 \%$ nos três primeiros anos (OHASHI et al., 2005). Essa atividade larval ocorre igualmente em brotos e ramos laterais adjacentes, especialmente quando a larva migra para esses locais, após o esgotamento das partes não lignificadas da madeira onde se encontra. Em casos extremos, de ataques intensos e repetitivos, pode ocorrer a morte do hospedeiro. Outras meliáceas suscetíveis, como o cedro (Cedrela odorata L.), sofrem o mesmo tipo de dano (CINTRON, 1990), por vezes, até com mais severidade que $S$. macrophylla (PAMPLONA et al., 1995). A árvore atacada tem a dominância apical quebrada (TAVERAS et al., 2004a) e apresenta poucos galhos, vários ponteiros co-dominantes e fuste com comprimento tão curto quanto mais repetidos forem os ataques, sendo comum o nível de infestação atingir $100 \%$ do plantio (CORNELIUS; WATT, 2003). Outros danos irreversíveis causados pela incidência do inseto são nós, bifurcações e tortuosidades no caule, que reduzem o valor comercial da madeira por afetarem sua trabalhabilidade e resistência mecânica, além de causarem perda parcial da tora (OHASHI et al., 2005).

Os primeiros sintomas da incidência de $H$. grandella são detectados na fase de viveiro, embora a fase de desenvolvimento de $S$. macrophylla cujo ataque mais compromete a planta, compreenda os primeiros três a seis anos. O grau de infestação é consideravelmente reduzido com a idade e crescimento da planta (SILVA, 1985), que adquire maior capacidade de recuperação ao ataque da praga a partir de aproximadamente seis metros de altura (HILJE; CORNELIUS, 2001). Há considerações sobre a aparente imunidade ao ataque em árvores adultas, embora haja registros de ataques desde viveiros a indivíduos com mais de $30 \mathrm{~m}$ de altura (VERGARA, 1997). O que ocorre é que a árvore é atacada durante todo seu desenvolvimento, sendo o ataque mais perceptível quando do período de formação do fuste, ou seja, nos primeiros anos de desenvolvimento. Esse longo período de suscetibilidade é preocupante, visto que prolonga o tempo necessário para monitoramento e a tomada de ações de controle nos plantios.

Atualmente, não existem estratégias para se controlar plenamente a incidência de $H$. grandella sobre meliáceas de importância comercial. Assim, esse trabalho tem por objetivo rever e atualizar aspectos bioecológicos das plantas hospedeiras e da criação do inseto, além de indicar as áreas de pesquisa mais promissoras para o controle de $H$. grandella em plantios de $S$. macrophylla .

\section{Aspectos bioecológicos}

A duração do ciclo biológico (ovo a adulto) de $H$. grandella é de aproximadamente 30 dias, dos quais o período larval compreende 20 dias e o período pupal, 10 dias, em temperatura de $30{ }^{\circ} \mathrm{C}$ (VARGAS et al., 2001; TAVERAS et al., 2004b). Uma única fêmea fertilizada pode pôr de 33 a 200 ovos durante três a quatro dias (GRIJPMA, 1971; BERTI FILHO, 1973; SARMENTO JUNIOR, 2001; OHASHI et al., 2002; SILVA, 2003) a até mais de 1.000 ovos em toda sua vida, sob condições de laboratório (GRIJPMA; ROBERTS, 1976).

H. grandella é associada com fatores sazonais. No Estado do Amazonas, a maior ocorrência de suas larvas em plantios de andiroba, Carapa guianensis Aubl., (Meliaceae), é durante a estação chuvosa (SILVA, 1985). Neste período, as plantas emitem novos ramos e brotos, com os quais há associação de $H$. grandella, conforme verificado em plantios de $S$. macrophylla no Peru (YAMAZAKI et al., 1990) e de C. odorata na Costa Rica (NEWTON et al., 1998). No Pará, foram registrados quatro ataques/planta/ano em plantios homogêneos de $S$. macrophylla (BATISTA, 2005; OHASHI et al., 2005).

Os adultos de Hypsipyla têm uma grande capacidade de vôo. As fềmeas são mais ativas nas duas primeiras noites após a emergência, quando provavelmente dispersam e localizam os hospedeiros (FASORANTI et al., 1982). Embora as mariposas tenham uma grande capacidade de dispersão, e de localização de hospedeiros isolados, elas permanecem na área atacada enquanto novas brotações ainda estiverem presentes, o que causa 
dano local severo (GRIFFITHS, 2001a). Os adultos são geralmente atraídos por árvores jovens, emitindo novas folhagens, e por árvores danificadas e com presença de fezes. Os ovos são colocados nas brotações, ramos ou folhas, geralmente na superfície dorsal das folhas (GRIJPMA e GARA, 1970; GRIFFITHS, 2001a).

As lagartas recém emergidas caminham sobre a superfície da planta antes de começarem a se alimentar, geralmente dentro de um ponto de crescimento ou de uma folha próxima ou axila de uma folha nova (GRIJPMA; GARA, 1970). As razões dessa movimentação no primeiro ínstar, que contribui para a alta mortalidade larval nessa fase, são desconhecidas. O local de alimentação da lagarta fica protegido por uma teia, contendo também fragmentos de planta e fezes. A pupação ocorre nos túneis de alimentação ou no solo ao redor da base da árvore (GRIFFITHS, 2001a).

O estudo da biologia e da ecologia de Hypsipyla spp. é dificultado pelo hábito noturno dos adultos, pela densidade populacional geralmente baixa, cuja mortalidade natural deve-se a fatores desconhecidos em quase metade dos casos, e pela dificuldade de acesso ao local de alimentação da lagarta e de criação desses insetos em laboratório (GRIFFITHS, 2001b; TAVERAS et al., 2004a). Pesquisas com monitoramento constante em áreas plantadas ou de ocorrência natural das plantas hospedeiras podem preencher tais lacunas do conhecimento.

\section{Plantas hospedeiras}

Espécies de Hypsipyla são relatadas se alimentando em plantas da família Meliaceae, subfamília Swietenioideae, que compreende 13 gêneros, a maioria dos quais servem como hospedeiros de Hypsipyla spp. (GRIFFITHS, 2001a). Estas preferem as Meliáceas endêmicas em seus respectivos centros de origem (CUNNINGHAM et al., 2005). No Brasil, ocorrem $H$. grandella em mogno, cedro e andiroba (Carapa guianensis Aubl.) (BERTI FILHO, 1973; MAUÉS, 2001; OHASHI et al., 2005) e $H$. ferrealis Hampson em frutos de andiroba (BECKER, 1973).

Outras partes da planta hospedeira também podem servir de sítios de desenvolvimento. A ausência de partes mais favoráveis na planta e/ou níveis populacionais elevados proporcionam ataques na casca (TAVERAS et al., 2004a) e até na raiz (YAMAZAKI et al., 1990), neste caso, podendo causar a morte da planta sem sintomas visíveis na parte aérea (PAMPLONA et al., 1995).

\section{Direcionamentos}

\section{Resistência da Planta Hospedeira}

É comum que se associem espécies de meliáceas exóticas introduzidas com a resistência à $H$. grandella. As espécies nativas $S$. macrophylla, C. guianensis e $C$. odorata são intensamente atacadas, enquanto as exóticas Khaya ivorensis A. Chev. (mogno africano), Toona ciliata M. J. Roem. (cedro australiano) e Azadirachta indica A. Juss. (nim) são resistentes. Entretanto, há um conflito de dados na literatura quando se considera a suposta certeza dessa associação com espécies congêneres (GRIJPMA, 1976; WATT et al., 2001). H. robusta ataca tanto meliáceas nativas de suas regiões de ocorrência natural ( $T$. ciliata e Chukrasia tabularis A. Juss, na Ásia e Austrália, e Khaya senegalensis (Desr.) A. Juss, na África) quanto espécies de outras regiões ( $S$. macrophylla e $C$. odorata, das Américas), embora com danos mais significativos nas espécies nativas (CUNNINGHAM et al., 2005). A substituição das espécies nativas por exóticas em plantios homogêneos deve ser considerada com cautela pelos riscos de adaptação de insetos-praga e patógenos nativos às essências introduzidas, principalmente se estas dispuserem de baixo ou limitado estoque de variabilidade genética. $\mathrm{O}$ uso de $K$. ivorensis em diferentes sistemas de plantio no Pará, em substituição à $S$. macrophylla, é muito difundido, a despeito da sua reduzida base genética. Nenhuma meliácea exótica usada em consórcios com $S$. macrophylla foi atacada por $H$. grandella nessa região (COSTA, 2000; OHASHI et al., 2002; BATISTA, 2005; OHASHI et al., 2005).

A estratégia de controle mais recomendada para H. grandella é a manipulação da resistência da planta hospedeira (CINTRON, 1990; NEWTON et al., 1993, 1999; FLOYD, 2001; TAVERAS et al., 2004a) pela formação de um banco ativo de germoplasma em sistemas agroflorestais ou silviculturais, seja por transferência de genes de resistência ou por resistência induzida. Poucos trabalhos têm sido conduzidos no sentido de se observar a variação genética da resistência intra e interespecífica. Diferentes procedências de $C$. odorata e $S$. macrophylla apresentaram variação na altura das árvores e na incidência de $H$. grandella na Costa Rica (NEWTON et al., 1999), mostrando a existência de não-preferência e tolerância como mecanismos de resistência das plantas hospedeiras, que podem ser trabalhadas na seleção de genótipos superiores (GRIJPMA, 1976; VALERA, 
1997). A partir desse ponto, o desenvolvimento de árvores mais resistentes seria um avanço substancial.

Testes de procedências e progênies, visando identificar variação genética em características como resistência à Hypsipyla spp., foram realizados em diferentes locais, com diferentes graus de sucesso (NEWTON et al., 1999; CORNELIUS; WATT, 2003; NAVARRO e HERNANDEZ, 2004; NAVARRO et al., 2004; WARD et al., 2008; WIGHTMAN et al., 2008). A identificação, por meio de biologia molecular, de strains genéticos de árvores resistentes ou tolerantes foi relatada por Novick et al. (2003).

No Brasil, estudos de variabilidade genética das plantas hospedeiras de $H$. grandella são recentes. Um sistema foi desenvolvido para caracterizar a estrutura genética de S. macrophylla (LEMES et al., 2002), e comprovou a existência de altos níveis de diversidade genética entre sete populações nativas de S. macrophylla distantes entre si na Amazônia brasileira, inclusive com diferenciação em escala local entre as populações (LEMES et al., 2003). Populações de S. macrophylla na América Central apresentaram correlação positiva entre as medidas de divergência genética e a distância geográfica entre as populações (NOVICK et al., 2003).

Não existem materiais de mogno selecionados para resistência à $H$. grandella. No entanto, o melhoramento visando à seleção de materiais tolerantes ou resistentes é importante para viabilizar a exploração comercial. Uma vez selecionados os materiais-elite, a clonagem destes possibilitará a manutenção de suas características. Para realizar a clonagem, a micropropagação apresenta vantagens, dentre elas, a rápida multiplicação de material em pequena área e em curto espaço de tempo, além do uso de outras técnicas no auxílio aos programas de melhoramento, inclusive das biotecnologias.

Há variação genética entre o cedro e o mogno em relação ao ataque de $H$. grandella, sendo que a tolerância parece ser o principal fator (WATT et al., 2001). Para os autores, a melhor opção para o manejo de $H$. grandella é a seleção de indivíduos resistentes e a aplicação de medidas silviculturais. Watt (2001) definiu prioridades nos trabalhos com resistência de plantas a Hypsipyla, relatando que mesmo níveis baixos de resistência podem ser importantes quando integrados com o controle biológico e outros métodos.

\section{Manejo silvicultural}

As boas práticas silviculturais são fundamentais para o manejo de $H$. grandella, sendo duas as práticas mais promissoras: a combinação de poda com seleção de sítios de alta qualidade e produção de mudas vigorosas em quantidade para replantios em áreas seguidamente atacadas; e o uso de espécies protetoras e que promovam sombra na fase inicial de crescimento, para reduzir a intensidade de ataque em plantios mistos (HAUXWELL, 2001). O autor cita também que são necessárias análises econômicas e dos benefícios dos tratamentos silviculturais utilizados, além da identificação de sistemas apropriados para cada região e análise do impacto desses sistemas nos inimigos naturais da praga.

Existem muitos experimentos com o objetivo de reduzir o dano de $H$. grandella por meio de diferentes técnicas silviculturais, cujos resultados são inconsistentes, devido a problemas como a falta de repetição experimental (HAUXWELL et al., 2001a). Os autores descrevem diversas técnicas silviculturais que podem ser promissoras no controle da praga e discutem quatro mecanismos essenciais de atuação dos tratamentos silviculturais: interferência na localização da planta hospedeira; redução da adequabilidade hospedeira; aumento de inimigos naturais; e recuperação da forma e incremento da altura da planta.

No Pará, Yared e Carpanezzi (1981) discorreram sobre duas estratégias de controle silvicultural de $H$. grandella em plantios de enriquecimento de mogno em vegetação secundária (método "recru"). A primeira seria promover um rápido estabelecimento e crescimento, para reduzir a fase de desenvolvimento da planta mais suscetível ao dano através da seleção dos melhores sítios e preparo do solo, luz sobre a copa e sombreamento lateral para promover o crescimento vertical; controle da matocompetição e poda de plantas atacadas. A segunda estratégia seria proteger as árvores do ataque da praga, com o plantio de baixas densidades de mogno por hectare, criando uma densa matriz com outras espécies arbóreas, maximizando assim a heterogeneidade florestal, com reprodução das condições da floresta natural.

No Amazonas, plantios de C. guianensis a pleno sol foram mais atacados por $\mathrm{H}$. grandella e tiveram menor desenvolvimento em diâmetro e altura que plantios mixados com vegetação de capoeira (SILVA, 1985). No Pará, os danos desse inseto foram mais pronunciados em plantios de $S$. macrophylla a pleno sol que em plantios mistos com a vegetação secundária (BRIENZAJUNIOR et al., 1990). S. macrophylla adquiriu uma resistência induzida quando consorciada com meliáceas resistentes à $H$. grandella (A. indica, K. ivorensis e T. ciliata), com 
ganho de $33 \%$ em altura em relação a plantas cultivadas em plantios homogêneos e a pleno sol; contudo, houve ataques generalizados nas plantas de $S$. macrophylla, em maior ou menor grau, independente dos sistemas de plantio utilizados (BATISTA, 2005).

Ensaios sobre avaliação dos efeitos do sombreamento são constantes e polêmicos, pois ao mesmo tempo em que alteram as condições ideais da planta para a oviposição e desenvolvimento de $H$. grandella, o que sugere a redução dos ataques em plantas sombreadas (YAMAZAKI et al., 1990; MAHROOF et al., 2002), retardam os ganhos de altura e diâmetro da árvore. Um regime com mais de $50 \%$ de sombreamento inibiu o crescimento de plantios de $C$. odorata e $C$. guianensis mixados à vegetação secundária no Pará, e favoreceu o aumento do número de ataques (OHASHI et al., 1993).

Consórcios de $S$. macrophylla com K. ivorensis, A. indica e T. ciliata proporcionaram eficiências de controle de $39 \%, 46 \%$ e $48 \%$, respectivamente, no Pará (BATISTA, 2005; OHASHI et al., 2005). Resultados ainda mais expressivos foram obtidos pela contagem diária de ovos de $H$. grandella em S. macrophylla consorciado, também no Pará (COSTA et al., 2000). A preferência para oviposição variou de $82 \%$ a $87 \%$ em $T$. ciliata, conforme o espaçamento, a despeito da resistência tipo antibiose que a planta possui em relação à $H$. grandella (OHASHI et al., 2000; OIANO NETO, 2000; SARMENTO JUNIOR, 2001; ALVES, 2002; BATISTA, 2005), cujas folhas proporcionam tempo letal médio $\left(\mathrm{TL}_{50}\right)$ de quase duas horas para larvas de primeiro ínstar a até oito minutos para as de terceiro ínstar (ALVES, 2002). Entretanto, o uso de árvoresarmadilha deve ser visto com cautela, pois há o risco da promoção do desenvolvimento do inseto na essência exótica. Além disso, a capacidade de oviposição de uma única fêmea é suficiente para que as posturas sejam, também, encontradas nas essências nativas restantes em quantidades suficientes para promover danos (GRIJPMA; ROBERTS, 1976). E há registros do decréscimo da eficiência desses sistemas isoladamente (COSTA, 2000).

A estratégia mais comum e eficiente para se recuperar o fuste reto da árvore atacada por $\mathrm{H}$. grandella é a poda dos ponteiros atacados (CORNELIUS, 2001), mas é questionável a viabilidade econômica desse método em larga escala. A aplicação de uma cola à base de polibuteno com piretróides no broto apical de $S$. macrophylla foi mais eficiente em relação à poda; contudo, esta aplicação é recomendada para pequenos plantios e, em casos de brotos já atacados, deve-se efetuar a poda dos mesmos (COSTA, 2000; OHASHI, 2002; OHASHI et al., 2002).

A enxertia com essências nativas suscetíveis ao inseto sobre exóticas é outra estratégia para o controle alternativo para conferir resistência induzida. Os constituintes químicos que conferem resistência à $T$. ciliata contra o ataque de $H$. grandella foram translocados à $C$. odorata sem obter, contudo, a mesma totalidade de ausência de ataques como ocorreu com a essência resistente (GRIJPMA; ROBERTS, 1976). A enxertia induziu a resistência das meliáceas exóticas para as espécies nativas $S$. macrophylla e $C$. odorata que, em uma escala de graus de resistência, foi classificada como moderada (SANTOS, 2001). Entretanto, enxertos de $S$. macrophylla sobre $A$. indica, T. ciliata e K. ivorensis não tiveram sucesso (BATISTA, 2005).

O manejo silvicultural é uma ação complementar no âmbito de um programa de Manejo Integrado de Pragas (MIP). Pesquisas nesse sentido devem objetivar o mínimo de ataque e o máximo de crescimento nas plantas. Áreas afins de pesquisa poderiam participar em consonância com a silvicultura em um plano de ação mais abrangente. O geoprocessamento, por exemplo, seria essencial na marcação e estudo da distribuição de árvores matrizes de diversas procedências em meio à ampla área de ocorrência natural de $S$. macrophylla e demais hospedeiros de $H$. grandella. Além disso, a análise da cobertura verde em plantios mistos ou homogêneos pode fornecer dados úteis para sistemas silviculturais ou agroflorestais.

\section{Uso de semioquímicos}

O uso de inseticidas convencionais tem sido desaconselhável para controle de $H$. grandella por razões como o hábito do inseto (críptico), a natureza dos danos (internos na planta), fatores climáticos (pluviosidade intensa na região de ocorrência natural) e o longo período de proteção necessário, que o torna oneroso, impraticável e danoso para o meio ambiente (WYLIE, 2001; MAHROOF et al., 2002). Apesar de alguns plantios em pequena escala mostrarem bons resultados com inseticidas (GOULET et al., 2005), tais produtos têm seu uso mais restrito a viveiros. Para tanto, várias técnicas alternativas vêm sendo estudadas, como o uso do feromônio sexual do inseto e de cairomônios das meliáceas (BOREK et al., 1991; SOARES et al., 2003; GOULET et al., 2005). 
Oiano Neto et al. (2000) e Silveira et al. (2002), estudando populações brasileiras de $H$. grandella, identificaram dois compostos nos extratos de fêmeas que mostraram resposta eletrofisiológica em antenas de machos por CG-EAD, Z9,E12-14:OH e Z9,E12-14:Ac. Não foi detectada a presença do composto acetato de hexadecenila e o Z3-16Ac não mostrou resposta eletrofisiológica. Testes de campo foram conduzidos com os quatro compostos sintéticos, em mistura ou individualmente, mas sem sucesso, o que indica que são necessários mais estudos para a obtenção de uma mistura do feromônio que atraia os machos de $\mathrm{H}$. grandella.

Espécies de Meliaceae distinguem-se pela ocorrência frequente de limonóides (SILVA et al., 1999). Embora sejam necessários bioensaios sobre a ação direta de limonóides isolados sobre $H$. grandella (AGOSTINHO et al., 1994), não foi possível esclarecer, puramente através desses compostos, a base da resistência natural (FERREIRA et al., 2005) ou induzida (PAULA et al., 1997, 1998). É provável que a resistência do tipo antibiose de $T$. ciliata a $H$. grandella seja devido à presença de esteróides pregnanos isolados das folhas (OIANO NETO, 2000), mas são precisos ensaios de avaliação nutricional para avaliação de seu potencial de controle e prováveis efeitos sobre $H$. grandella. Há vários estudos de identificação dos compostos presentes em plantas de mogno, cedro e Toona (PAULA et al., 1997; MAIA et al., 2000; SOARES et al., 2003; LAGO et al., 2006), mas esses estudos ainda não são conclusivos, sobre qual ou quais compostos presentes na três espécies de plantas seriam os responsáveis pela atração de $H$. grandella, e nem os responsáveis pela resistência de T. ciliata.

\section{Controle biológico}

Embora Hypsipyla spp. seja atacada por uma grande diversidade de inimigos naturais artrópodes, não há redução suficiente da abundância larval e dos danos verificados em níveis aceitáveis em plantações de meliáceas (BLANCO-METZLER et al., 2001; SANDS; MURPHY, 2001).

Estudos detalhados da dinâmica populacional e de tabelas de vida de espécies de Hypsipyla em suas regiões de origem podem identificar agentes de controle biológico potenciais (SANDS; HAUXWELL, 2001; SANDS; MURPHY, 2001). Pouco se conhece sobre os parasitóides de ovos de Hypsipyla spp., uma área considerada prioritária para a pesquisa. Como são de difícil localização no campo, a exposição de ovos produzidos em laboratório pode servir como meio de capturar parasitóides para identificação e avaliação (SANDS; HAUXWELL, 2001).

Contudo, sempre deve haver um plano de ação voltado para a descoberta de novos agentes de controle biológico (TAVERAS et al., 2004a) que possam ter especificidade significativa com o inseto, principalmente quando se considera a grande biodiversidade ainda desconhecida no ecossistema amazônico. Ataques de Synoeca surinama L. (Hymenoptera: Vespidae) e percevejos sobre lagartas, e de vespas (Chalcididae) e moscas (Tachinidae) sobre pupas foram observados em plantios de $C$. guianensis (SILVA, 1985). Um nematóide, duas vespas e um fungo (Beauveria sp.) foram observados sobre ovos e lagartas (YAMAZAKI et al., 1990). No Pará, Trichogrammatomyia tortricis Girault (Hymenoptera: Trichogrammatidae) incidiu em $26,5 \%$ de ovos de $H$. grandella em plantio de S. macrophylla e em, aproximadamente, $45 \%$ dos ovos quando em plantios consorciados desta essência com $K$. ivorensis e T. ciliata (BATISTA, 2005; OHASHI et al., 2005).

Hypsipyla spp. são alvos difíceis para o controle microbiano, principalmente porque suas lagartas são crípticas e ocorrem em baixas densidades populacionais. Foram relatados diversos entomopatógenos atacando Hypsipyla spp., como fungos, nematóides e microsporídeos, além de patógenos de outros lepidópteros, como Bacillus thuringiensis (HAUXWELL et al., 2001b). Como estratégias promissoras para controle de Hypsipyla, os autores citam a introdução para controle por longo período e a liberação inundativa com inseticidas microbianos.

\section{Considerações Finais}

Vários são os trabalhos visando ao controle de $H$. grandella em meliáceas, com diferentes graus de sucesso, em diferentes países, envolvendo controle químico, biológico, silvicultural, resistência de plantas, além de métodos integrados de controle. No entanto, nenhuma técnica isolada tem demonstrado resultado satisfatório em campo, sendo necessário desenvolver práticas dentro do conceito de MIP, que consiste na combinação harmônica de vários métodos de controle para manter a praga em níveis que não causem dano econômico, tendo como base, o controle biológico natural, levando-se em conta os aspectos econômicos, sociais e ambientais. Um bom programa de manejo integrado deve fundamentar-se no conhecimento de aspectos bioecológicos da praga e 
das árvores hospedeiras, visando estabelecer critérios confiáveis de tomada de decisão e enfatizar as práticas preventivas, priorizando o melhoramento genético, o uso de práticas silviculturais adequadas, o controle biológico e o controle comportamental (HILJE; CORNELIUS, 2001).

As áreas prioritárias de pesquisa para o manejo integrado de Hypsipyla spp. são: busca e emprego de material genético de alta qualidade; estabelecimento de métodos adequados para produção de mudas sadias; seleção de sítios para promover um crescimento vigoroso das plantas; promoção de crescimento inicial por meio do uso de fertilizantes, combate a ervas daninhas, condução de um fuste reto e poda; incorporação de material de plantio resistente sob regimes silviculturais apropriados, enfatizando o uso de espécies mistas; desenvolvimento de medidas de controle emergenciais, como controle químico localizado; aplicação inundativa de inimigos naturais e de técnicas de monitoramento para detectar picos populacionais; e geração de informações técnicas de extensão e serviços para os agricultores (SPEIGHT, 2001).

A despeito da importância de Hypsipyla spp. em limitar a produção comercial de algumas espécies de meliáceas, muitos aspectos da taxonomia, biologia e ecologia dessas espécies ainda são desconhecidos. São fundamentais os estudos de populações de diferentes regiões geográficas e de diferentes hospedeiros e partes da planta, utilizando-se exames morfológicos, técnicas moleculares e bioquímicas. Quanto à ecologia, é necessário conhecer melhor a capacidade de vôo e a dispersão dos adultos, bem como a localização do hospedeiro e o movimento larval (GRIFFITHS, 2001b).

Pesquisas de longo prazo devem ser incentivadas por governos e órgãos de fomento para o manejo silvicultural, controle biológico e uso de semioquímicos de $H$. grandella, além da resistência natural de $S$. macrophylla. Testes comparativos padronizados devem possibilitar comparações com outras pesquisas e evitar duplicidade de esforços através de redes de pesquisadores com aderência ao tema.

Apesar de o Brasil possuir a maior área de reservas naturais de $S$. macrophylla, as pesquisas sobre o controle de $H$. grandella são poucas e devem ser incentivadas, principalmente, aquelas que associem a variabilidade genética das procedências de $S$. macrophylla com a sua suscetibilidade à $H$. grandella.

\section{Referências}

AGOSTINHO, S. M. M.; SILVA, M. F. G. F.; FERNANDES, J. B.; VIEIRA, P. C.; PINHEIRO, A. L.; VILELA, E. F. Limonoids from Toona ciliata and speculations on their chemosystematic and ecological significance. Biochemical Systematics and Ecology, Oxford, v. 22, n. 3, p. 323-328, 1994.

ALVES, M. Z. N. Efeito letal de Toona ciliata M. J. Roem, folhas maduras de mogno (Swietenia macrophylla King) e seus extratos à lagarta de Hypsipyla grandella Zeller em laboratório. 2002. 53 f. Dissertação (Mestrado em Agronomia), Instituto de Ciências Agrárias, Faculdade de Ciências Agrárias do Pará.

BATISTA, T. F. C. Resistência induzida ao mogno brasileiro Swietenia macrophylla King por meliáceas resistente no controle da broca Hypsipyla grandella Zeller, $1848 \mathrm{em}$ consórcio e em sistema agroflorestal. 2005. 81 f. Tese (Doutorado em Ciências Agrárias) - Universidade Federal Rural da Amazônia, Belém, PA.

BECKER, V. O. Estudios sobre el barrenador Hypsipyla grandella (Zeller) Lepidoptera, Pyralidae. XVI. Observaciones sobre la biologia de $H$. ferrealis (Hampson), una especie afin. Turrialba, v. 23, p. 154-161, 1973.

BERTI FILHO, E. Observações sobre a biologia de Hypsipyla grandella (Zeller, 1848) (Lepidoptera, Phycitidae). 1973. $108 \mathrm{f}$ Dissertação (Mestrado) - Escola Superior de Agricultura Luiz de Queiroz, Universidade de São Paulo, Piracicaba

BLANCO-METZLER, H.; VARGAS, C.; HAUXWELL, C. Indigenous parasitoids and exotic introductions for the control of Hypsipyla grandella (Zeller) (Lepidoptera: Pyralidae) in Latin America. In: FLOYD, R. B.; HAUXWELL, C. (Ed.) Hypsipyla Shoot Borers in Meliaceae: Proceedings of an International Workshop. Canberra: Australian Centre for International Agricultural Research. 2001, p. 140-145.

BOREK, V.; KALINOVA, B.; VALTEROVA, I.; HOCHMUT, R.; VROKOC, J. Sex pheromone gland volatiles from Hypsipyla grandella females. Acta Entomologica Bohemoslovaca, Praga, v. 88, n. 181-186, 1991.

BRIENZA JUNIOR, S.; CASTRO, T. C. A.; VIANA, L. M. Ensaio de espécies florestais sob duas condições ecológicas: 1. Avaliações silviculturais. In: Congresso Florestal Brasileiro, 6. São Paulo. Anais... Sociedade Brasileira de Silvicultura, 1990, p. 616-624.

CINTRON, B. B. Cedrela odorata L. Cedro hembra, Spanish cedar. In: BURNS, R. M.; HONKALA, B. H. (Ed.) Silvics of North America: 2. Hardwoods. Washington: USDA Forest Service, 1990. p. 250-257 (Agricultural Handbook, 654).

CORNELIUS, J. P. The effectiveness of pruning in mitigating Hypsipyla grandella attack on young mahogany (Swietenia macrophylla King) trees. Forest Ecology and Management, Amsterdam, n. 148, p. 287-289, 2001. 
CORNELIUS, J. P.; WATT, A. D. Genetic variation in a Hypsipyla-attacked clonal trial of Cedrela odorata under two pruning regimes. Forest Ecology and Management, Amsterdam, n. 183, p. $341-349,2003$.

COSTA, M. S. S. Controle de Hypsipyla grandella Zeller (broca do mogno) utilizando a planta resistente Toona ciliata Roem (cedro australiano) e os métodos mecânico e cultural no plantio de Swietenia macrophylla King (mogno). 2000. 52 f. Dissertação (Mestrado em Ciências Florestais), Instituto de Ciências Agrárias, Faculdade de Ciências Agrárias do Pará.

COSTA, M. S. S.; OHASHI, O. S.; SILVA, J. N. M.; SILVA, M. F. G. F. Controle da broca do mogno através da preferência de ovoposição de Hypsipyla grandella Zeller sobre Toona ciliata M. J. Roem. plantado em consórcio com Swietenia macrophylla King. Belém: Embrapa Amazônia Oriental. 2000, 3 p. (Comunicado Técnico, 47).

CUNNINGHAM, S. A.; FLOYD, R. B.; GRIFFITHS, M. W.; WYLIE, F. R. Patterns of host use by the shoot-borer Hypsipyla robusta (Pyralidae: Lepidoptera) comparing five Meliaceae tree species in Asia and Australia. Forest Ecology and Management, Amsterdam, n. 205, p. 351-357, 2005.

FASORANTI, J. O.; GARA, R. I.; GEISZLER, D. R. Laboratory studies on the flight capacity of the mahogany shoot borer, Hypsipyla grandella (Zeller) (Lepidoptera, Pyralidae). Zeitschrift für Angewandte Entomologie, Hamburg, v. 93, p.182-186, 1982.

FERREIRA, I. C. P.; CORTEZ, D. A. G.; SILVA, M. F. G. F.; RODRIGUES FILHO, E.; VIEIRA, P. C.; FERNANDES, J. B. Acylperoxylated and seco-mexicanolides from stems of Khaya anthotheca. Journal of Natural Products, n. 68, p. 413-416, 2005.

FLOYD, R. B. International Workshop on Hypsipyla shoot borers in Meliaceae: general conclusions and research priorities. In: FLOYD, R. B.; HAUXWELL, C. (Ed.) Hypsipyla Shoot Borers in Meliaceae: Proceedings of an International Workshop. Canberra: Australian Centre for International Agricultural Research. 2001, p. 183-187.

GOULET, E.; RUEDA, A.; SHELTON, A. Management of the mahogany shoot borer, Hypsipyla grandella (Zeller) (Lepidoptera: Pyralidae), through weed management and insecticidal sprays in 1- and 2-year-old Swietenia humilis Zucc. plantations. Crop Protection, Amsterdam, n. 24, p. 821-828, 2005

GRIFFITHS, M. W. The biology and host relations of the red cedar tip moth, Hypsipyla robusta, in Australia. In: SNELL, A.; VIZE, S. (Ed.) Opportunities for the New Millennium: Proceedings of the Australian Forest Growers Biennial Conference. Canberra: Australian Forest Growers. 2000, p. 135-140.

GRIFFITHS, M. W. The biology and ecology of Hypsipyla shoot borers. In: FLOYD, R. B.; HAUXWELL, C. (Ed.) Hypsipyla Shoot Borers in Meliaceae: Proceedings of an International Workshop. Canberra: Australian Centre for International Agricultural Research. 2001, p. 74-80.
GRIFFITHS, M. W. Taxonomy, biology and ecology of Hypsipyla spp. In: FLOYD, R. B.; HAUXWELL, C. (Ed.) Hypsipyla Shoot Borers in Meliaceae: Proceedings of an International Workshop. Canberra: Australian Centre for International Agricultural Research. 2001, p. 85.

GRIJPMA, P. Studies on the shootborer Hypsipyla grandella Zeller. V. Observations on a rearing technique and on host selection behavior of adults in captivity. Turrialba, San Jose, n. 21, p. 202-213, 1971.

GRIJPMA, P. Resistance of Meliaceae against the shoot borer Hypsipyla with particular reference to Toona ciliata M. J. Roem. var. australis (F. V. Muell.) C. DC. In: WHITMORE, J. L. (Ed.) Studies on the shootborer Hypsipyla grandella (Zeller) Lep. Pyralidae. Vol. III. San José: Centro Agronómico Tropical de Investigación y Enseñanza. 1976, p. 90-96 (Miscellaneous Publications, 101).

GRIJPMA, P.; GARA, R. I. Studies on the shootborer Hypsipyla grandella (Zeller): II. host preference of the larva. Turrialba, San Jose, n. 20, p. 241-247, 1970.

GRIJPMA, P.; ROBERTS, S. C. Biological and chemical ascreening for the basis of resistance of Toona ciliata M. J. Roem. var australis. In: WHITMORE, J. L. (Ed.) Studies on the shootborer Hypsipyla grandella (Zeller) Lep. Pyralidae. Vol. III. San José: Centro Agronómico Tropical de Investigación y Enseñanza. 1976, p. 102-109 (Miscellaneous Publications, 101).

HAUXWELL, C. Silvicultural management of Hypsipyla spp. In: FLOYD, R. B.; HAUXWELL, C. (Ed.) Hypsipyla Shoot Borers in Meliaceae: Proceedings of an International Workshop. Canberra: Australian Centre for International Agricultural Research. 2001, p. 166.

HAUXWELL, C.; MAYHEW, J.; NEWTON, A. Silvicultural management of Hypsipyla species. In: FLOYD, R. B.;

HAUXWELL, C. (Ed.) Hypsipyla Shoot Borers in Meliaceae:

Proceedings of an International Workshop. Canberra:

Australian Centre for International Agricultural Research. 2001a, p. $151-163$.

HAUXWELL, C.; VARGAS, C.; FRIMPONG, E. O. Entomopathogens for control of Hypsipyla spp. In: FLOYD, R. B.; HAUXWELL, C. (Ed.) Hypsipyla Shoot Borers in Meliaceae: Proceedings of an International Workshop. Canberra: Australian Centre for International Agricultural Research. 2001b, p. 131-139.

HILJE, L.; CORNELIUS, J. Es inmanejable Hypsipyla grandella como plaga forestal? Manejo Integrado de Plagas, Turrialba, $n$. 61, p. 1-4, 2001.

LAGO, J. H. G.; SOARES, M. G.; BATISTA-PEREIRA, L. G.; SILVA, M. G. F.; CORRÊA, A. G.; FERNANDES, J. B.; VIEIRA, P. C.; ROQUE, N. F. Volatile oil from Guarea macrophylla ssp. tuberculata: seasonal variation and electroantennographic detection by Hypsipyla grandella. Phytochemistry, Amsterdam, n. 67 , p. 589-594, 2006.

LEMES, M. R.; BRONDANI, R. P. V.; GRATTAPAGLIA, D. Multiplexed systems of microsatellite markers for genetic analysis of mahogany, Swietenia macrophylla King. Journal of Heredity, Oxford, n. 93, p. 287-291, 2002. 
LEMES, M. R.; GRIBEL, R.; PROCTOR, J.; GRATTAPAGLIA, D. Population genetic structure of mahogany (Swietenia macrophylla King, Meliaceae) across the Brazilian Amazon, based on variation at microsatellite loci: implications for conservation. Molecular Ecology, Vancouver, n. 12, p. 2875-2883, 2003.

MAHROOF, R. M.; HAUXWELL, C.; EDIRISINGHE, J. P.; WATT, A. D.; NEWTON, A. C. Effects of artificial shade on attack by the mahogany shoot borer, Hypsipyla robusta (Moore). Agricultural and Forest Entomology, Midlothian, n. 4, p. 283-292, 2002.

MAIA, B. H. L. N. S.; PAULA, J. R.; SANT'ANA, J.; SILVA, M. F. G. F.; FERNANDES, J. B.; VIEIRA, P. C.; COSTA, M. S. S.; OHASHI, O. S.; SILVA, J. N. M. Essential oils of Toona and Cedrela species (Meliaceae): taxonomic and ecological implications. Journal of Brazilian Chemical Society, São Paulo, n. 11, p. 629-639, 2000.

MAUÉS, M. M. A review of Hypsipyla grandella Zeller research in Pará State, Brazil. In: FLOYD, R. B.; HAUXWELL, C. (Ed.) Hypsipyla Shoot Borers in Meliaceae: Proceedings of an International Workshop. Canberra: Australian Centre for International Agricultural Research. 2001b, p. 63-66.

NAIR, K. S. S. Tropical Forest Insect Pests: Ecology, Impact and Management. New York: Cambridge University Press. 2007, 404 p.

NAVARRO, C.; HERNÁNDEZ, G. Progeny test analysis and population differentiation of mesoamerican mahogany (Swietenia macrophylla). Agronomía Costarricense, San Jose, v. 28, p. 37-51, 2004.

NAVARRO, C.; MONTAGNINI, F.; HERNÁNDEZ, G. Genetic variability of Cedrela odorata Linnaeus: results of early performance of provenances and families from Mesoamerica grown in association with coffee. Forest Ecology and Management, Amsterdam, n. 192, p. 217-227, 2004

NEWTON, A. C.; BAKER, P.; RAMNARINE, S.; MESEN, J. F.; LEAKEY, R. R. B. The mahogany shoot-borer, prospects for control. Forest Ecology and Management, Amsterdam, n. 57, p. 301-328, 1993.

NEWTON, A. C.; CORNELIUS, J. P.; MESÉN, J. F.; COREA, E. A.; WATT, A. D. Variation in attack by the mahogany shoot borer, Hypsipyla grandella (Lepidoptera: Pyralidae), in relation to host growth and phenology. Bulletin of Entomological Research, Cambridge, n. 88, p. 319-326, 1998.

NEWTON, A. C.; WATT, A. D.; LOPEZ, F.; CORNELIUS, J. P.; MESÉN, J. F.; COREA, E. A. Genetic variation in host susceptibility to attack by the mahogany shoot borer, Hypsipyla grandella (Zeller). Agricultural and Forest Entomology, Midlothian, n. 1, p. 11-18, 1999.

NOVICK, R. R.; DICK, C. W.; LEMES, M. R.; NAVARRO, C.; CACCONE, A.; BERMINGHAM, E. Genetic structure of mesoamerican populations of big-leaf mahogany (Swietenia macrophylla) inferred from microsatellite analysis. Molecular Ecology, Vancouver, n. 12, p. 2885-2893, 2003.

OHASHI, O. S. Inseticida para salvar o mogno. Pesquisa FAPESP, São Paulo, n. 74, p. 32-33, 2002.
OHASHI, S. T.; COSTA, L. G. S.; PEDROSO, L. M.

Enriquecimento de floresta tropical mecanicamente explorada com as espécies Cedrela odorata L. (cedro) e Carapa guianensis Aubl. (andiroba), no planalto de Curuá-Una, Pará, Brasil. Belém: Faculdade de Ciências Agrárias do Pará, 1993. (Boletim da FCAP, 21).

OHASHI, O. S.; COSTA, M. S. S.; SILVA, J. N. M.; SILVA, M. F. G. F. Resistência do tipo antibiose apresentada pelas folhas novas de Toona ciliata M. J. Roem às lagartas de Hypsipyla grandella Zeller. Belém: Embrapa Amazônia Oriental. 2000, 3 p. (Comunicado Técnico, 48).

OHASHI, O. S.; SILVA, J. N. M.; SILVA, M. F. G. F.; COSTA, M. S. S.; SARMENTO JUNIOR, R. G.; SANTOS, E. B.; ALVES, M. Z. N.; PESSOA, A. M. C.; SILVA, T. C. O.; BITTENCOURT, P. R. G.; BARBOSA, T. C.; SANTOS, T. M. Manejo integrado da broca do mogno Hypsipyla grandella Zeller (Lep. Pyralidae). In: POLTRONIERI, L. S.; TRINDADE, D. R. (Ed.) Manejo integrado das principais pragas e doenças de cultivos amazônicos, Belém: Embrapa Amazônia Oriental. 2002, p. 91-120.

OHASHI, O. S.; SILVA JUNIOR, M. L.; LAMEIRA, O. A.; SILVA, J. N. M.; LEÃO, N. V. M.; TEREZO, E. F.; BATISTA, T. F. C.; HIDAKA, D. Z. L.; ALMEIDA, G. B.; BITTENCOURT, P. R. G.; GOMES, F. S.; NEVES, G. A. M. Danos e controle de broca Hypsipyla grandella em plantios de mogno Swietenia macrophylla no Estado do Pará. In: POLTRONIERI, L. S.; TRINDADE, D. R.; SANTOS, I. P. (Ed.) Pragas e doenças de cultivos amazônicos. Belém: Embrapa Amazônia Oriental. 2005, p. $125-144$.

OIANO NETO, J. Estudo fitoquímico de Toona ciliata: uma contribuição à quimiossistemática do gênero e à ecologia da interação Hypsipyla-Meliaceae. 2000. 287 f. Tese (Doutorado em Química), Departamento de Química, Universidade Federal de São Carlos.

OIANO NETO, J.; BRAGA, P. A. C.; SILVEIRA, N.; PEREIRA, L. B.; SANT'ANA, J.; SILVA, M. F. G. F.; CORREA, A. G.; FERNANDES, J. B.; VIEIRA, P. C.; OHASHI, O. S. Sex pheromone of Hypsipylla grandella and essential oils of Toona ciliata, Cedrela odorata, Switenia macrophyla, Carapa guianensis and Cabraleae canjerana (Meliaceae). In: IUPAC INTERNATIONAL SYMPOSIUM ON THE CHEMISTRY OF NATURAL PRODUCTS, 22., 2000, São Carlos. Natural products on the last biological frontier: abstracts. [S.1]: IUPAC, 2000. PPA-028.

PAMPLONA, A. M. S. R.; ANDREAZZE, R.; AZEVÊDO, C. P.; LIMA, R. M. B. Registro de danos nas raízes do mogno (Swietenia macrophylla King). Manaus: Embrapa Amazônia Ocidental. 1995, 3 p. (Pesquisa em andamento, 19).

PAULA, J. R.; VIEIRA, I. J. C.; SILVA, M. F. G. F.; RODRIGUES FILHO, E.; FERNANDES, J. B.; VIEIRA, P. C.; PINHEIRO, A. L.; VILELA, E. F. Sesquiterpenes, triterpenoids, limonoids and flavonoids of Cedrela odorata graft and speculations on the induced resistance against Hypsipyla grandella. Phytochemistry, Amsterdam, n. 44, p. 1449-1454, 1997. 
PAULA, J. R.; CASTRO-GAMBOA, I.; OIANO NETO, J.; SILVA, M. F. G. F.; RODRIGUES FILHO, E.; FERNANDES, J. B.; VIEIRA, P. C.; PINHEIRO, A. L. Chemistry of Cedrela odorata graft and speculations on the induced resistance against Hypsipyla grandella. Anais da Academia Brasileira de Ciências, Rio de Janeiro, n. 70, p. 737-742, 1998.

SANDS, D. P. A.; HAUXWELL, C. Discussion summary. Biological control of Hypsipyla spp. In: FLOYD, R. B.; HAUXWELL, C. (Ed.) Hypsipyla Shoot Borers in Meliaceae: Proceedings of an International Workshop. Canberra: Australian Centre for International Agricultural Research. 2001b, p. 146-147.

SANDS, D. P. A.; MURPHY, S. T. Prospects for biological control of Hypsipyla spp. with insect agents. In: FLOYD, R. B.; HAUXWELL, C. (Ed.) Hypsipyla Shoot Borers in Meliaceae: Proceedings of an International Workshop. Canberra: Australian Centre for International Agricultural Research. 2001b, p. 121-130.

SANTOS, E. B. Estímulos produzidos por Swietenia macrophylla (mogno), Cedrela odorata (cedro) e Toona ciliata (cedro vermelho) e resistência induzida pela enxertia de $S$. macrophylla e $\boldsymbol{C}$. odorata sobre T. ciliata, Khaya ivorensis e Azadirachta indica à lagarta de Hypsipyla grandella. 2001. 26 f. Dissertação (Mestrado em Agronomia), Instituto de Ciências Agrárias, Universidade Federal Rural da Amazônia.

SARMENTO JUNIOR, R. G. Biologia de Hypsipyla grandella (Zeller, 1848) e avaliação da resistência de Swietenia macrophylla King, Cedrela odorata L., Toona ciliata Roem e Toona sp. à broca das meliáceas em laboratório. 2001. 60 f. Dissertação (Mestrado em Agronomia), Instituto de Ciências Agrárias, Faculdade de Ciências Agrárias do Pará.

SILVA, N. M. Características biológicas e demográficas de Hypsipyla grandella (Zeller, 1848) (Pyralidae, Lepidoptera) e níveis de infestação sob dois sistemas de plantio de Carapa guianensis Aubl. (Meliaceae) no Amazonas. 1985. 103 f. Dissertação (Mestrado em Ciências Biológicas), Departamento de Ciências Fundamentais e Desenvolvimento Agrícola, Instituto Nacional de Pesquisas da Amazônia.

SILVA, M. F. G. F.; AGOSTINHO, S. M. M.; PAULA, J. R.; OIANO NETO, J.; CASTRO-GAMBOA, I.; RODRIGUES FILHO, E.; FERNANDES, J. B.; VIEIRA, P. C. Chemistry of Toona ciliata and Cedrela odorata Graft (Meliaceae): chemosystematic and ecological significance. Pure and Applied Chemistry, Praga, n. 71, p. 1083-1087, 1999.

SILVA, T. C. O. Dieta artificial para a criação de Hypsipyla grandella (Zeller, 1848) (Lepidoptera), broca das meliáceas, em laboratório. 2003. 73 f. Dissertação (Mestrado em Agronomia), Instituto de Ciências Agrárias, Universidade Federal Rural da Amazônia.

SILVEIRA, N. A.; PEREIRA, L. G. B.; CORRÊA, A. G.; SILVA, M. F. G. F.; FERNANDES, J. B.; VIEIRA, P. C.

Síntese dos componentes do feromônio da broca-do-cedro, Hypsipyla grandella. In: REUNIÃO ANUAL DA SOCIEDADE BRASILEIRA DE QUÍMICA, 25., 2002. Resumos... Poços de Caldas: SBQ, 2002. 1 CD-ROM. Ref. QO-093.
SOARES, M. G.; BATISTA-PEREIRA, L. G.; FERNANDES, J. B.; CORRÊA, A. G.; SILVA, M. F. G. F.; VIEIRA, P. C.; RODRIGUES FILHO, E.; OHASHI, O. S. Electrophysiological responses of female and male Hypsipyla grandella (Zeller) to Swietenia macrophylla essential oils. Journal of Chemical Ecology, New York, n. 29, p. 2143-2151, 2003.

SPEIGHT, M. R. Integrated Pest Management of Hypsipyla spp. - Discussion summary. In: FLOYD, R. B.; HAUXWELL, C. (Ed.) Hypsipyla Shoot Borers in Meliaceae: Proceedings of an International Workshop. Canberra: Australian Centre for International Agricultural Research. 2001, p. 179.

TAVERAS, R.; HILJE, L.; HANSON, P.; MEXZÓN, R.; CARBALLO, M.; NAVARRO, C. Population trends and damage patterns of Hypsipyla grandella (Lepidoptera: Pyralidae) in a mahogany stand, in Turrialba, Costa Rica. Agricultural and Forest Entomology, Midlothian, n. 6, p. 89-98, 2004a.

TAVERAS, R.; HILJE, L.; CARBALLO, M. Development of Hypsipyla grandella (Zeller) (Lepidoptera: Pyralidae) in response to constant temperatures. Neotropical Entomology, Londrina, n. 33, p. 1-6, 2004b.

VALERA, F. P. Recursos geneticos de Swietenia y Cedrela en los neotropicos: Propuestas para acciones coordinadas. Roma: Food and Agriculture Organization. 1997, 60 p.

VARGAS, C.; SHANNON, P. J.; TAVERAS, R.; SOTO, F.; HILJE, L. Un nuevo método para la cría masiva de Hypsipyla grandella. Manejo Integrado de Plagas, Turrialba, n. 62, p. 1-4, 2001.

VERGARA, A. J. B. Aproximación hacia un manejo integrado del barrenador de las Meliaceas, Hypsipyla grandella (Zeller). Revista Forestal Venezolana, Mérida, n. 41, p. 23-28, 1997.

WARD, S. E.; WIGHTMAN, K. E.; SANTIAGO, B. R. Early results from genetic trials on the growth of Spanish cedar and its susceptibility to the shoot borer moth in the Yucatan Peninsula, Mexico. Forest Ecology and Management, Amsterdam, v. 255, p. 356-364, 2008.

WATT, A. D. Host plant resistance for control of Hypsipyla spp. In: FLOYD, R. B.; HAUXWELL, C. (Ed.) Hypsipyla Shoot Borers in Meliaceae: Proceedings of an International Workshop. Canberra: Australian Centre for International Agricultural Research. 2001, p. 106.

WATT, A. D.; NEWTON, A. C.; CORNELIUS, J. P. Resistance in mahoganies to Hypsipyla species - A basis for Integrated Pest Management. In: FLOYD, R. B.; HAUXWELL, C. (Ed.) Hypsipyla Shoot Borers in Meliaceae: Proceedings of an International Workshop. Canberra: Australian Centre for International Agricultural Research. 2001, p. 89-95.

WIGHTMAN, K. E.; WARD, S. E.; HAGGAR, J. P.; SANTIAGO, B. R.; CORNELIUS, J. P. Performance and genetic variation of big-leaf mahogany (Swietenia macrophylla King) in provenance and progeny trials in the Yucatan Peninsula of Mexico. Forest Ecology and Management, Amsterdam, v. 255, p. 346-355, 2008. 
WYLIE, F. R. Control of Hypsipyla spp. shoot borers with chemical pesticides: a review. In: FLOYD, R. B.; HAUXWELL, C. (Ed.) Hypsipyla Shoot Borers in Meliaceae: Proceedings of an International Workshop. Canberra: Australian Centre for International Agricultural Research. 2001, p. 109-117.

YAMAZAKI, S.; TAKETANI, A.; FUJITA, K.; VASQUES, C.; IKEDA, T. Ecology of Hypsipyla grandella and its seasonal changes in population density in Peruvian Amazon forest. Japan Agricultural Research Quarterly, Tokyo, n. 24, p. 149-155, 1990 .
YARED, J. A. G.; CARPANEZZI, A. A. Conversão de capoeira alta da Amazônia em povoamento de produção madeireira: o método 'recrú' e espécies promissoras. Belém: Embrapa Amazônia Oriental. 1981, 27 p. (Boletim de Pesquisa, 25).

Recebido em 06 de março de 2009 e aprovado em 14 de dezembro de 2009 
